\title{
EQUAÇÕES DE ALTURA, VOLUME E AFILAMENTO PARA EUCALIPTO E ACÁCIA ESTABELECIDOS EM SISTEMA SILVIPASTORIL
}

\author{
Marcelo Dias Müller ${ }^{1}$, Thiago Taglialegna Salles ${ }^{2}$, Domingos Sávio Campos Paciullo ${ }^{1}$, Alexandre Magno \\ Brighenti ${ }^{1}$, Carlos Renato Tavares de Castro ${ }^{1}$ \\ ${ }^{1}$ Eng. Florestal, Dr., EMBRAPA Gado de Leite, Juiz de Fora, MG, Brasil - marcelo.muller@embrapa.br; \\ domingos.paciullo@embrapa.br; alexandre.brighenti@embrapa.br; carlos.castro@embrapa.br \\ ${ }^{2}$ Eng. Florestal, Doutorando em Ciências Florestais, UFV, Viçosa, MG, Brasil - thiagotsalles@ gmail.com \\ Recebido para publicação: 26/08/2013 - Aceito para publicação: 21/03/2014
}

\begin{abstract}
Resumo
O objetivo deste trabalho foi selecionar modelos alométricos para estimativa de altura, volume e afilamento do tronco de duas espécies florestais estabelecidas em um sistema silvipastoril com 10 anos de idade. Para a variável volume, foram testados modelos de simples e dupla entrada e para o afilamento do tronco foram testados modelos segmentados e não segmentados. Para estimativa da altura das árvores de eucalipto, foi selecionado o modelo semilogarítmico e para a acácia o modelo de melhor desempenho foi o exponencial. Entre os modelos volumétricos, aqueles de dupla entrada apresentaram melhores ajustes, sendo que o modelo de Schumacher \& Hall se destacou com estatísticas ligeiramente superiores aos demais. Para o afilamento de tronco, os modelos segmentados apresentaram melhor desempenho. Para árvores de eucalipto, o modelo de Max \& Burkhart se mostrou mais adequado, e para acácia, o modelo de Parresol foi o que apresentou o melhor ajuste. De forma geral, os modelos se ajustaram melhor aos dados das árvores de eucalipto.

Palavras-chave: Agrossilvicultura; modelagem; programa ABC.
\end{abstract}

\begin{abstract}
Height, volume and taper equations for eucalipt and acacia mangium established in a silvopastoral system. The objective of this work was to select allometric models for height, volume and taper estimation of two forestry species established in a ten years old silvopastoral system. Single (breast height diameter as the independent variable) and double entry (breast height diameter and total height as independent variables) models were evaluated for the volume estimation. We evaluated segmented and non-segmented models for the taper equations. The semilogaritmic model and the exponential model fitted better for the estimation of eucalyptus and acacia trees height, respectively. In general, the double entry volumetric models presented the best adjustments and Schumacher \& Hall's model presented slightly higher statistics among the models. In the case of taper models, we observed that the segmented models fitted better to the data for both species. Max \& Burkhar's model and the Parresol's model were selected for eucalyptus trees and acacia trees, respectively. In general, all tested models fitted better to eucalyptus trees.

Keywords: Agroforestry; Modeling; Eucalyptus; Acacia.
\end{abstract}

\section{INTRODUÇÃO}

Os sistemas silvipastoris, caracterizados por tecnologias de uso da terra em que atividades pecuárias e florestais são integradas em uma mesma área, de forma simultânea ou escalonadas no tempo (NAIR, 1993; MACEDO, 2000), têm chamado a atenção de cientistas, técnicos e produtores rurais, como uma alternativa mais sustentável de uso da terra. Essa integração de componentes proporciona uma série de benefícios ambientais, econômicos e sociais, conforme relatam diversos autores (XAVIER et al., 2003; BODDEY et al., 2004; NEVES et al., 2004; NEVES et al., 2009; MÜLLER et al., 2011).

Em 2010 foi lançado pelo governo federal, o programa ABC (Agricultura de Baixo Carbono), visando o cumprimento de compromisso voluntário para redução de emissões de carbono, assumido na $15^{\text {a }}$ Conferência das Partes da Convenção Quadro das Nações Unidas sobre Mudanças Climáticas (COP 
15), ocorrida em Copenhague, Dinamarca. Esse programa estabelece as regras para financiamento a projetos de recuperação de áreas degradadas, plantio direto, fixação biológica de nitrogênio e integração lavoura-pecuária-floresta (ou agrossilvipastoril).

Entretanto, existem ainda algumas lacunas no conhecimento a serem trabalhadas pela pesquisa, a fim de subsidiar a elaboração, o planejamento e a adoção desses sistemas. Conforme relatam Salles et al. (2012), são importantes as avaliações econômicas, para subsidiar os agentes de financiamento, os técnicos e os produtores envolvidos nesse tipo de investimento, e a análise da viabilidade desse tipo de projeto, que está vinculada às estimativas de produção desses sistemas. Para tanto, faz-se necessário o uso de ferramentas para a estimação da produção florestal nesses sistemas.

A modelagem de equações alométricas para estimação de variáveis dendrométricas em povoamentos estabelecidos em monocultivo é um assunto bastante avançado. Entretanto, em se tratando de sistemas agrossilvipastoris, onde os espaçamentos utilizados são mais amplos, ainda são escassos os estudos de modelagem de equações para estimação de parâmetros dendrométricos.

Nogueira et al. (2008) destacam que o espaçamento inicial de plantio interfere na forma do fuste, o que está diretamente ligado ao rendimento na conversão em madeira serrada de árvores de pinus e eucalipto. Nesse estudo, os autores observaram uma tendência de aumento na conicidade dos fustes em árvores de Pinus taeda L. com o aumento do espaçamento entre plantas.

Destacam-se alguns estudos, como, por exemplo, os de Fontan et al. (2011), que estudaram o crescimento de clones de eucalipto estabelecidos em sistemas agroflorestais, submetidos a diferentes tratamentos de desrama. Os autores ajustaram o modelo de Schumacher (logaritmizado), na forma simples (em função da idade), para predição de diâmetro, altura e volume. Silveira et al. (2011) ajustaram modelos de altura e afilamento do tronco para árvores de Eucalyptus camaldulensis Dehn., estabelecidas nas mesmas condições, visando a classificação de árvores para postes. Salles et al. (2012) estudaram o uso do modelo de Clutter para predição do crescimento e produção de clones de eucalipto estabelecidos em sistemas agroflorestais e Binoti et al. (2012) estudaram formas da função Weibull e hiperbólica para descrição e predição da distribuição diamétrica na mesma área. É importante mencionar que todos esses trabalhos foram desenvolvidos nas mesmas condições de espaçamento, no noroeste de Minas Gerais.

Dada a importância dos estudos de modelagem específicos, e de posse de dados de inventários realizados em sistema silvipastoril na região da Zona da Mata Mineira, o objetivo deste trabalho foi ajustar e comparar modelos alométricos para estimação de altura, volume e afilamento do tronco (taper) para árvores de Eucalyptus grandis Hill ex Maiden e Acacia mangium Willd.

\section{MATERIAL E MÉTODOS}

Os dados analisados neste estudo provêm de um sistema agrossilvipastoril misto com dez anos de idade implantado em uma área de quatro hectares, no município de Coronel Pacheco. A área é montanhosa de Latossolo Vermelho-Amarelo com baixa fertilidade natural, localizada na região da Zona da Mata, MG.

O clima da região é do tipo Cwa, mesotérmico, apresentando verão quente e chuvoso e inverno frio e seco. A pluviosidade média anual situa-se ao redor de $1.500 \mathrm{~mm}$, distribuídos irregularmente, e somente $13 \%$ desse total ocorrem durante os meses de maio a setembro, considerado como o período da seca. A temperatura média anual é de $18{ }^{\circ} \mathrm{C}$ nos meses mais frios e $22^{\circ} \mathrm{C}$ durante o verão (PACIULLO et al., 2011).

O sistema foi implantado em novembro de 1997, sendo estabelecidas faixas de árvores em curva de nível, compostas por quatro linhas, com espaçamento de $3 \times 3 \mathrm{~m}$ entre plantas, intercaladas por faixas de pastagem com largura aproximada de 30 metros, totalizando 342 árvores por hectare. Nas faixas de árvores, foram plantadas, alternadamente, mudas de Eucalyptus grandis e Acacia mangium, propagadas por sementes, totalizando assim 171 árvores de cada espécie por hectare.

Aos sete anos de idade, foi realizado o desbaste seletivo das árvores de eucalipto, reduzindo a população a 60 árvores por hectare.

Dada a competição com o eucalipto, a mortalidade entre as árvores de acácia foi significativa, o que resultou em uma população de 45 árvores por hectare, aos 10 anos.

A área foi dividida em oito piquetes que foram manejados em sistema de pastejo rotativo, com períodos de ocupação variando entre cinco e sete dias e período de descanso entre 35 e 49 dias, em função da época do ano (período chuvoso e seco respectivamente).

Foram medidos os diâmetros a 1,3 m (DAP) de 239 árvores de eucalipto e de 177 árvores de 
acácia. A partir destes dados, foi feita a caracterização da distribuição diamétrica do povoamento em classes de $5 \mathrm{~cm}$ e seleção de 3 árvores-amostra por classe diamétrica para cubagem rigorosa. Também foi estimada a altura total $(\mathrm{Ht})$ e realizado cubagem rigorosa de 27 árvores de eucalipto e de 17 árvores de acácia, empregando-se um pentaprisma de Weller e um suunto. As árvores foram cubadas segundo o método de Smalian, considerando-se o comprimento de seção de 1,5 m.

A partir dos dados de cubagem utilizando as variáveis diâmetro a 1,3 $\mathrm{m}$ de altura (DAP) e altura total (Ht), foram ajustadas equações hipsométricas referentes aos seguintes modelos:

$\begin{array}{lll}01 & H t=e^{\beta_{0}+\beta_{1} D A P^{-1}} & \text { (Exponencial) } \\ 02 & H t=e^{\beta_{0}+\beta_{1} \ln D A P} & \text { (Potência) } \\ 03 & H t=\beta_{0}+\beta_{1} D A P^{-2} & \text { (Hiperbólico 1) } \\ 04 & H t=\beta_{0}+\beta_{1} D A P & \text { (Polinômio) } \\ 05 & H t=D A P^{2}\left(\beta_{0} D A P+\beta_{1} D A P^{2}\right)^{-1} & \text { (Hiperbólico 2) } \\ 06 & H t=\beta_{0}+\beta_{1} \ln D A P & \text { (Semilogarítmico) } \\ 07 & H t=\beta_{0} e^{-e^{\beta_{1}-\beta_{2} D A P}} & \text { (Sigmoidal) }\end{array}$

Considerando a tendência de crescimento diferenciado, bem como de formação de fustes com maior conicidade em espaçamentos maiores, como é o caso de sistemas silvipastoris (COUTO et al., 1977; BERGER et al., 2002; NOGUEIRA et al., 2008), optou-se por comparar, para o ajuste de equações de volume (V), modelos de simples entrada com modelos de dupla entrada (CAMPOS; LEITE, 2009), sendo:

Dupla entrada

\begin{tabular}{|c|c|c|}
\hline 08 & $V=\beta_{0} D A P^{\beta_{1}} H t^{\beta_{2}}$ & (Schumacher \& Hall) \\
\hline 09 & $V=e^{\beta_{0}+\beta_{1} \ln \left(D A P^{2} H t\right)}$ & (Spurr logaritmizado) \\
\hline 10 & $V=\beta_{0}+\beta_{1}\left(D A P^{2} H t\right)$ & (Spurr) \\
\hline
\end{tabular}

Simples entrada

$$
\begin{aligned}
& V=e^{\beta_{0}+\beta_{1} \ln D A P+\beta_{2} D A P^{-1}} \\
& V=\beta_{0}+\beta_{1} D A P^{2} \\
& V=\beta_{0}+\beta_{1} D A P+\beta_{2} D A P^{2}
\end{aligned}
$$

(Brenac)

(Koperzky e Gehrhardt)

13

(Hohenadl \& Kreen)

Da mesma forma, foram comparados modelos segmentados com modelos não segmentados, para o ajuste das equações de taper, conforme as equações a seguir:

Não segmentados

$$
d c c_{i}=D A P\left\{\beta_{0}+\beta_{1} \ln \left[1-\lambda X^{1 / 3}\right]\right\} \text { (Biging) }
$$

$$
d c c_{i}=10^{\beta_{0}} D A P^{\beta_{1}}\left(H t-h_{i}\right)^{\beta_{2}} H t^{\beta_{3}}
$$

(Demaerschalk)

16

$$
d c c_{i}=D A P \beta_{0}\left[1+\beta_{1} \ln \left(1-\beta_{2} X^{\beta_{3}}\right)\right]
$$

(Garay)

$$
d c c_{i}=D A P\left(\beta_{0}+\beta_{1} X+\beta_{2} X^{2}\right)^{1 / 2} \quad \text { (Kozak) }
$$


Segmentados

18

$d c c_{i}=D A P\left[\beta_{0}(X-1)+\beta_{1}\left(X^{2}-1\right)+\beta_{2}\left(a_{1}-X\right)^{2} I_{1}+\beta_{3}\left(a_{2}-X\right)^{2} I_{2}\right]^{1 / 2}$ (Max \& Burkhart)

19

$$
d c c_{i}=D A P\left\{Z^{2}\left(\beta_{0}+\beta_{1} Z\right)+\left(Z-a_{1}\right)^{2}\left[\beta_{2}+\beta_{3}\left(Z+2 a_{1}\right)\right] I\right\}^{1 / 2},
$$

(Parresol)

em que: $\mathrm{i}=$ seção do fuste;

$\mathrm{h}_{\mathrm{i}}=$ altura na seção $\mathrm{i}$;

dcc $_{\mathrm{i}}=$ diâmetro com casca na seção i;

$\lambda=\left(1-e^{-\beta_{0} / \beta_{1}}\right)$;

$X=h_{i} / H t$

$I_{i}=\left[\begin{array}{l}=1 \mathrm{se} X \leq a_{i} \\ =0 \operatorname{se} X>a_{i}\end{array} ;\right.$

$a_{i}=$ ponto de ligação dos polinômios;

$Z=1-h_{i} / H t ; \quad I=\left[\begin{array}{l}=1 \mathrm{se} Z \leq a_{1} \\ =0 \operatorname{se} Z>a_{1}\end{array}\right.$

A seleção dos modelos foi feita com base na análise gráfica de resíduos e no erro padrão das estimativas $\left(S_{y x}\right)$ e do coeficiente de determinação ajustado $\left(R^{2}\right)$, calculado segundo a metodologia proposta por Kvålseth (1985), conforme as equações abaixo:

$$
S_{y x}=\sqrt{\left.\sum_{i=1}^{n}\left(Y_{i}-\hat{Y}\right)^{2}\right)^{2} /(n-k)}
$$

$$
\bar{R}^{2}(\%)=100\left[1-(n-1 / n-p-1)\left(1-R^{2}\right)\right.
$$

em que: $\mathrm{Y}=$ valor observado;

$\hat{Y}=$ valor estimado;

$\mathrm{n}=$ número de observações;

$\mathrm{k}=$ número de parâmetros do modelo;

$R^{2}=1-\sum_{i=1}^{n}\left(Y_{i}-\hat{Y}_{i}\right)^{2} / \sum_{i=1}^{n}\left(Y_{i}-\bar{Y}_{i}\right)^{2}$

$\mathrm{p}=$ número de variáveis independentes.

\section{RESULTADOS E DISCUSSÃO}

\section{Modelos hipsométricos}

Os coeficientes e as estatísticas referentes aos modelos hipsométricos ajustados e testados para as duas espécies são apresentados na tabela 1 .

Para o eucalipto, o $R^{2}$ variou de 0,89 a 0,69 e para acácia a variação foi entre 0,91 e 0,80 . Esses resultados contrariam aqueles apresentados por Barros et al. (2004) e Machado et al. (2011). Esses autores observaram que, em plantios de Pinus oocarpa, tanto o avanço da idade quanto o maior número de desbastes, o que implica redução na densidade de árvores por hectare (e, portanto, cria uma situação de arranjo espacial das árvores similar àquela encontrada em sistemas silvipastoris), ocasionaram descaracterização da relação hipsométrica, com redução gradativa do $R^{2}$. Segundo esses autores, as alturas tendem a se homogeneizar, independentemente da distribuição diamétrica.

Considerando os critérios $R^{2}$ e $S_{y x}$, os modelos que tiveram melhor desempenho ao se estimar altura tanto de eucalipto quanto de acácia no sistema silvipastoril foram os de equação números 01 (exponencial) e 06 (semilogarítmica). Os piores resultados foram obtidos pelas equações 03 (hiperbólica 1) e 04 (polinômio).

Para verificar se os modelos foram de fato adequados, foi realizada a análise gráfica de resíduos para os modelos 01 e 06, os quais apresentaram melhor ajuste (Figura 1). 
Tabela 1. Parâmetros estatísticos das equações ajustadas para estimação de altura total de árvores de Eucalyptus grandis e Acacia mangium, aos 10 anos de idade, estabelecidas em sistema silvipastoril.

Table 1. Statistics parameters of hipsometric equations modelling for total height estimation of Eucalyptus grandis and Acacia mangium trees established in a 10 year-old silvopastoral system.

\begin{tabular}{|c|c|c|c|c|c|}
\hline Equação & $\hat{\beta}_{0}$ & $\hat{\beta}_{1}$ & $\hat{\beta}_{2}$ & $R^{2}$ & $S_{y x}$ \\
\hline \multicolumn{6}{|c|}{ Eucalipto } \\
\hline 01 & 3,62695 & $-12,24641$ & - & 0,88 & 2,24 \\
\hline 02 & 1,29173 & 0,55306 & - & 0,87 & 2,32 \\
\hline 03 & 26,42336 & $-1.380,08123$ & - & 0,69 & 3,58 \\
\hline 04 & 9,01286 & 0,47506 & - & 0,84 & 2,61 \\
\hline 05 & 0,61691 & 0,02034 & - & 0,88 & 2,19 \\
\hline 06 & $-14,98192$ & 11,61892 & - & 0,89 & 2,14 \\
\hline 07 & 31,92605 & 0,65009 & 0,06759 & 0,87 & 2,31 \\
\hline \multicolumn{6}{|c|}{ Acácia } \\
\hline 01 & 2,98045 & $-6,25504$ & - & 0,91 & 0,84 \\
\hline 02 & 1,36274 & 0,42429 & - & 0,87 & 1,05 \\
\hline 03 & 15,47883 & $-378,36072$ & - & 0,85 & 1,13 \\
\hline 04 & 7,16637 & 0,32111 & - & 0,80 & 1,28 \\
\hline 05 & 0,51620 & 0,04443 & - & 0,90 & 0,90 \\
\hline 06 & $-2,20652$ & 5,46311 & - & 0,90 & 0,91 \\
\hline 07 & 16,30010 & 0,61231 & 0,13953 & 0,89 & 0,97 \\
\hline
\end{tabular}
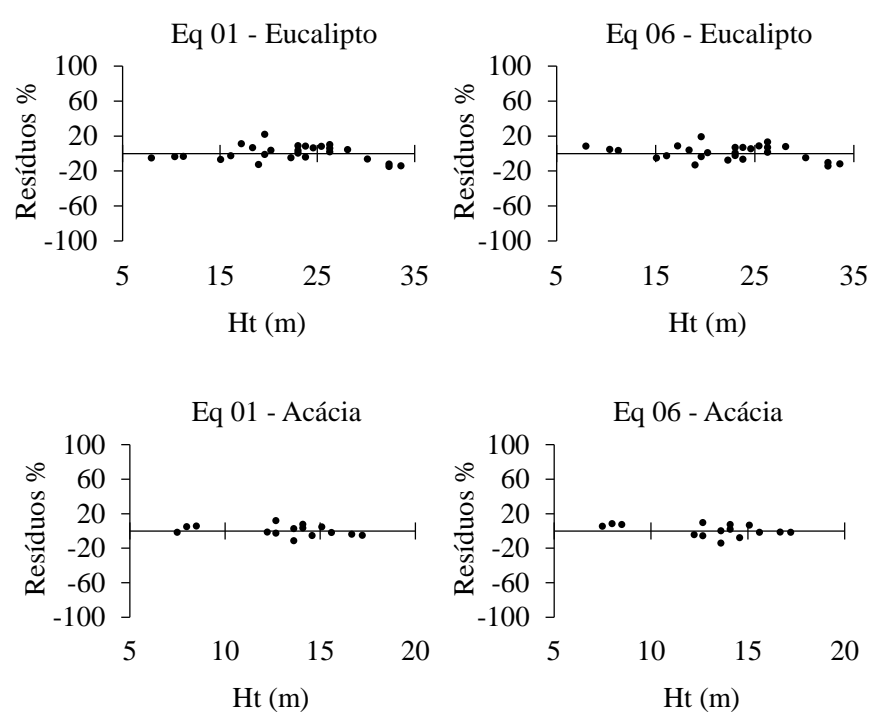

Figura 1. Gráficos de resíduos (em \%) das estimativas de altura em função do DAP de árvores de Eucalyptus grandis e Acacia mangium, aos 10 anos de idade, estabelecidas em sistema silvipastoril.

Figure 1. Graphs of residues (\%) of the estimations of height as a function of DBH of Eucalyptus grandis and Acacia mangium trees established in a 10 year-old silvopastoral system.

Os dois modelos selecionados apresentaram comportamentos semelhantes na análise gráfica de resíduos, em que se verificou ausência de tendências na estimativa das alturas e dispersão homogênea dos resíduos com amplitude de erro de +/- 20\%, tanto para o eucalipto quanto para a acácia.

\section{Modelos volumétricos}

$\mathrm{Na}$ tabela 2 , são apresentados os coeficientes e as estatísticas dos modelos volumétricos ajustados e testados para ambas as espécies.

De modo geral, os modelos de dupla entrada geraram estimativas mais precisas. Os modelos volumétricos ajustados apresentaram $R^{2}$ variando entre 0,97 e 0,95 , para o eucalipto e entre 0,89 e 0,78 
para a acácia, com destaque para o modelo de dupla entrada 08 (Schumacher \& Hall), que apresentou estimativas de maior exatidão ligeiramente superiores aos demais para as duas espécies.

Segundo Campos e Leite (2009), o modelo de Schumacher \& Hall tem sido o mais difundido por apresentar, normalmente, melhores ajustes com estimativas não tendenciosas. Os autores ainda observam que o modelo de Spurr também tem sido bastante difundido, em função da maior facilidade do ajuste. Para a acácia, o modelo de Spurr na sua forma logaritmizada apresentou as piores estimativas. Veiga et al. (2000) obtiveram bons resultados com o modelo de Schumacher \& Hall, na forma logaritmizada, para acácia. Entretanto, os autores observaram que o modelo de Meyer (não logarítmico) apresentou os melhores ajustes. Schneider et al. (1997) utilizaram o modelo de Spurr para estimação de volume de árvores de Eucalyptus dunii Maiden. O modelo foi escolhido em função da presença do termo $\mathrm{d}^{2} \mathrm{~h}$ na equação, que explica melhor a paraboloide característica da forma daquelas árvores. Da mesma forma Tonini e Schwengber (2006) selecionaram as equações de Schumacher \& Hall e Spurr.

Tabela 2. Parâmetros estatísticos das equações para estimação de volume total de árvores de Eucalyptus grandis e Acacia mangium, aos 10 anos de idade, estabelecidas em sistema silvipastoril.

Table 2. Statistics parameters of hipsometric equations modelling for total height estimation of Eucalyptus grandis and Acacia mangium trees established in a 10 year-old silvopastoral system.

\begin{tabular}{|c|c|c|c|c|c|}
\hline Equação & $\hat{\beta}_{0}$ & $\hat{\beta}_{1}$ & $\hat{\beta}_{2}$ & $R^{2}$ & $S_{y x}$ \\
\hline \multicolumn{6}{|c|}{ Eucalipto } \\
\hline 08 & 0,00005 & 2,26242 & 0,56836 & 0,97 & 0,14 \\
\hline 09 & 0,00729 & 0,00003 & - & 0,96 & 0,15 \\
\hline 10 & $-10,25667$ & 0,99792 & - & 0,96 & 0,15 \\
\hline 11 & $-9,71231$ & 2,70333 & 6,40454 & 0,96 & 0,16 \\
\hline 12 & $-0,13630$ & 0,00106 & - & 0,95 & 0,17 \\
\hline 13 & 0,19500 & $-0,02761$ & 0,00153 & 0,96 & 0,16 \\
\hline \multicolumn{6}{|c|}{ Acácia } \\
\hline 08 & 0,09762 & 3,35862 & $-3,63268$ & 0,89 & 0,04 \\
\hline 09 & 0,02900 & 0,00002 & - & 0,78 & 0,06 \\
\hline 10 & $-8,15801$ & 0,74017 & - & 0,81 & 0,06 \\
\hline 11 & 6,01467 & $-1,35389$ & $-73,41315$ & 0,85 & 0,05 \\
\hline 12 & 0,00500 & 0,00039 & - & 0,83 & 0,05 \\
\hline 13 & $-0,05860$ & 0,00771 & 0,00020 & 0,83 & 0,06 \\
\hline
\end{tabular}

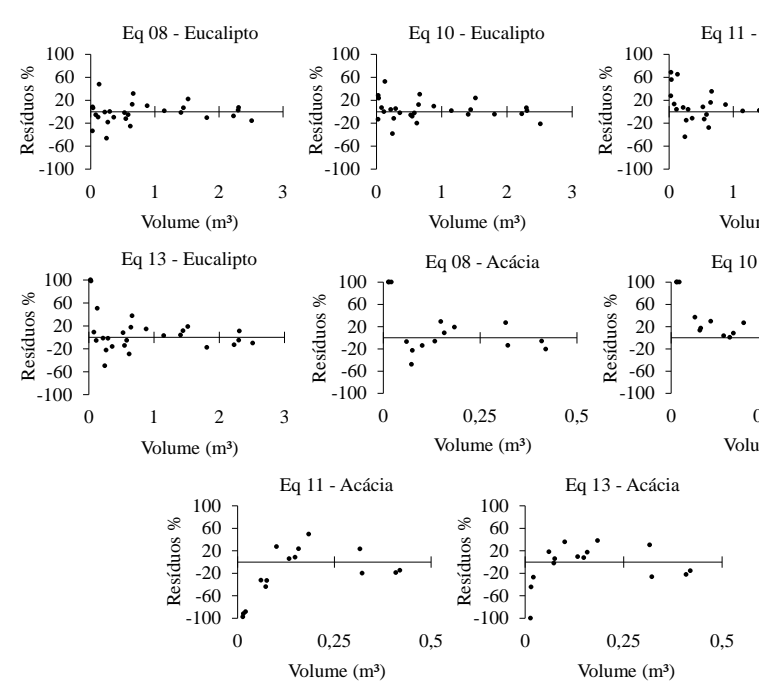

Figura 2. Gráficos de resíduos (em \%) das estimativas de volume em função de DAP e Ht para os modelos de dupla entrada (Eq 08 e 10) e de simples entrada (Eq 11 e 13) de árvores de Eucalyptus grandis e de Acacia mangium.

Figure 2. Graphs of residues (\%) of the estimations of volume as a function of DBH and Total Height for double entry models (Eq 08 and 10) and simple entry (Eq 11 and 13) of Eucalyptus grandis and Acacia mangium trees established in a 10 year-old silvopastoral system. 
Dentre os modelos de simples entrada, destacam-se os modelos de equação número 11 (Brenac) e 13 (Hohenadl \& Kreen). Barros e Silva Júnior (2009) obtiveram resultados semelhantes com as equações de Brenac, Shumacher \& Hall e Spurr, para espécies de uma florestal tropical densa na região Amazônica.

Pela análise gráfica de resíduos, pode-se observar que a amplitude de erro da estimativa tende a aumentar para árvores com menor volume (Figura 2).

\section{Modelos de afilamento do tronco}

Na tabela 3 são apresentados os coeficientes e as estatísticas dos modelos volumétricos ajustados e testados para ambas as espécies.

Tabela 3. Parâmetros estatísticos da modelagem de equações de afilamento de árvores de Eucalyptus grandis e Acacia mangium, aos 10 anos de idade, estabelecidas em sistema silvipastoril.

Table 3. Statistics parameters of taper equations modelling of Eucalyptus grandis and Acacia mangium trees established in a 10 year-old silvopastoral system.

\begin{tabular}{|c|c|c|c|c|c|c|c|c|}
\hline Equação & $\hat{\beta}_{0}$ & $\hat{\beta}_{1}$ & $\hat{\beta}_{2}$ & $\hat{\beta}_{3}$ & $\mathrm{a}_{1}$ & $\mathrm{a}_{2}$ & $R^{2}$ & $S_{y x}$ \\
\hline \multicolumn{9}{|c|}{ Eucalipto } \\
\hline 14 & 1,25377 & 0,98248 & - & - & - & - & 0,97 & 2,14 \\
\hline 15 & 0,17139 & 1,00310 & 1,41175 & $-1,52341$ & - & - & 0,95 & 2,67 \\
\hline 16 & 1,22309 & 1,56467 & 0,46864 & 0,43916 & - & - & 0,92 & 2,11 \\
\hline 17 & 0,94450 & $-2,05708$ & 1,11280 & - & - & - & 0,91 & 3,49 \\
\hline 18 & $-1,29685$ & 0,63688 & 62,66899 & 1,72169 & 0,089 & 0,44 & 0,97 & 2,11 \\
\hline 19 & 0,45164 & 0,45333 & $-459,78880$ & 179,83144 & 0,850 & & 0,97 & 2,14 \\
\hline \multicolumn{9}{|c|}{ Acácia } \\
\hline 14 & 1,26787 & 0,81399 & - & - & - & - & 0,91 & 2,57 \\
\hline 15 & 0,42470 & 0,92159 & 1,38614 & $-1,61982$ & - & - & 0,91 & 2,77 \\
\hline 16 & 1,25233 & 2,78723 & 0,29064 & 0,50297 & - & - & 0,93 & 2,53 \\
\hline 17 & 1,42106 & $-3,85911$ & 2,52459 & - & - & - & 0,84 & 3,40 \\
\hline 18 & $-1,53557$ & 0,71266 & $-105,72250$ & 70,52480 & 0,090 & 0,15 & 0,92 & 2,56 \\
\hline 19 & 2,69340 & $-3,61227$ & $-10,21641$ & 9,72511 & 0,480 & & 0,93 & 2,44 \\
\hline
\end{tabular}

Os modelos ajustados apresentaram elevados valores para $R^{2}$, variando entre 0,97 e 0,91 para eucalipto e entre 0,93 e 0,84 para acácia. De maneira geral, os modelos segmentados de Max \& Burkhart \& Parresol (equações 18 e 19) geraram os melhores resultados. Dentre os modelos não segmentados, destacou-se o modelo de Biging (equação 14), entretanto, os modelos de Demaerschalk e de Garay (equações 15 e 16) também se mostraram eficientes em estimar o perfil do tronco.

Nesse sentido, Souza et al. (2008a) observaram que os modelos não segmentados Garay e Biging geraram bons ajustes $\left(R^{2}>98,0 \%\right)$ para árvores de eucalipto com 16 anos de idade em espaçamento de 3 x 3 metros. Para modelos segmentados ajustados aos mesmos dados, Souza et al. (2008b) observaram que o modelo de Max \& Burkhart apresentou os melhores ajustes. Entretanto, segundo os mesmos autores, o modelo de Parresol foi o que se mostrou menos eficiente, contrariando os resultados deste trabalho.

A figura 3 apresenta as análises gráficas de resíduos para as equações que melhor representaram o afilamento de tronco das espécies estudadas.

A análise dos gráficos de dispersão mostra que os modelos se apresentam bastante semelhantes dentro de cada espécie. Para o eucalipto, é observada leve tendência a subestimar em diâmetros em torno de $35 \mathrm{~cm}$. Já para a acácia, os modelos tendem a apresentar resíduos mais distantes da linha de regressão em árvores com DAP acima de $25 \mathrm{~cm}$.

A qualidade dos ajustes dos modelos melhor classificados para cada tipo de modelagem pode ser vista nas figuras 4,5 e 6 , que ilustram as tendências das estimativas geradas pelas equações diante dos dados observados.

Na figura 4 é possível observar que, para o eucalipto, os dois modelos selecionados tendem a superestimar a altura em árvores com diâmetros acima de $40 \mathrm{~cm}$. Já para a acácia não é observado esse efeito. No caso da variável volume (Figura 5), os modelos selecionados para eucalipto não apresentaram tendenciosidade, ao passo que para a acácia se observa tendência de dispersão em árvores com diâmetro 
acima de $25 \mathrm{~cm}$. As curvas referentes às equações de volume de dupla entrada 08 e 10 (Figura 5) foram geradas com um valor médio de altura igual a $27 \mathrm{~m}$ para eucalipto e $15 \mathrm{~m}$ para acácia. Para a tendência de perfil (Figura 6) se observa o mesmo fenômeno, ou seja, os modelos selecionados apresentaram menor tendenciosidade para o eucalipto.

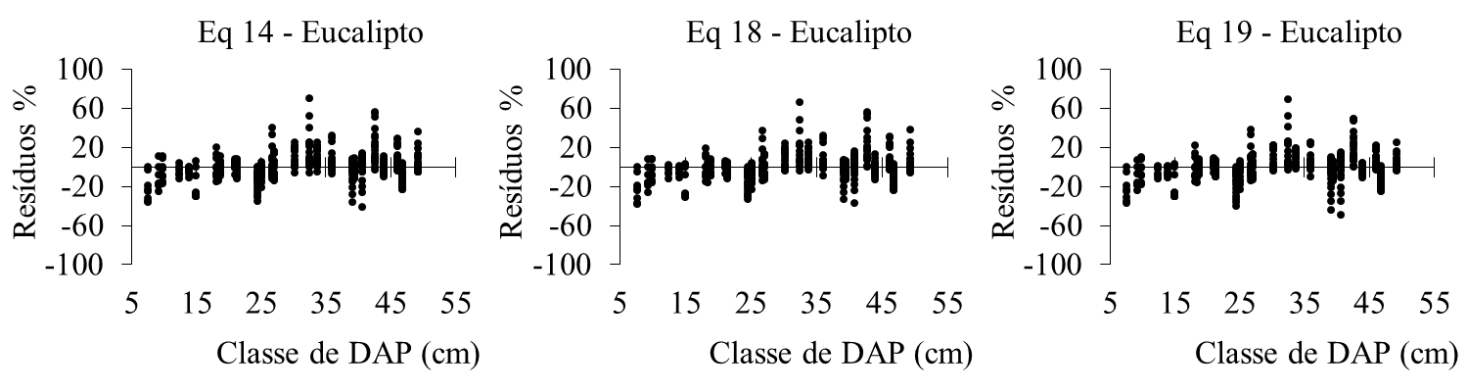

Eq 14 - Acácia

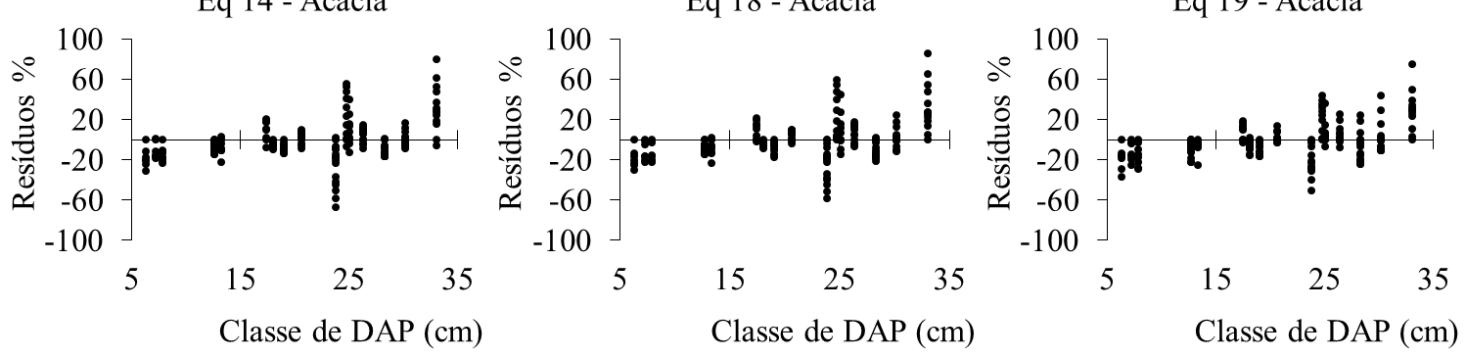

Figura 3. Gráficos de resíduos (em \%) das estimativas de diâmetro com casca ao longo do tronco geradas para árvores de Eucalyptus grandis e de Acacia mangium.

Figure 3. Graphs of residues (\%) of the estimations of diameter of Eucalyptus grandis and Acacia mangium trees established in a 10 year-old silvopastoral system.

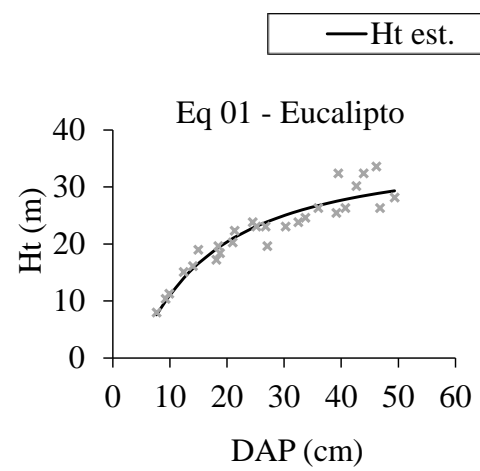

\section{$\times$ Ht obs.}
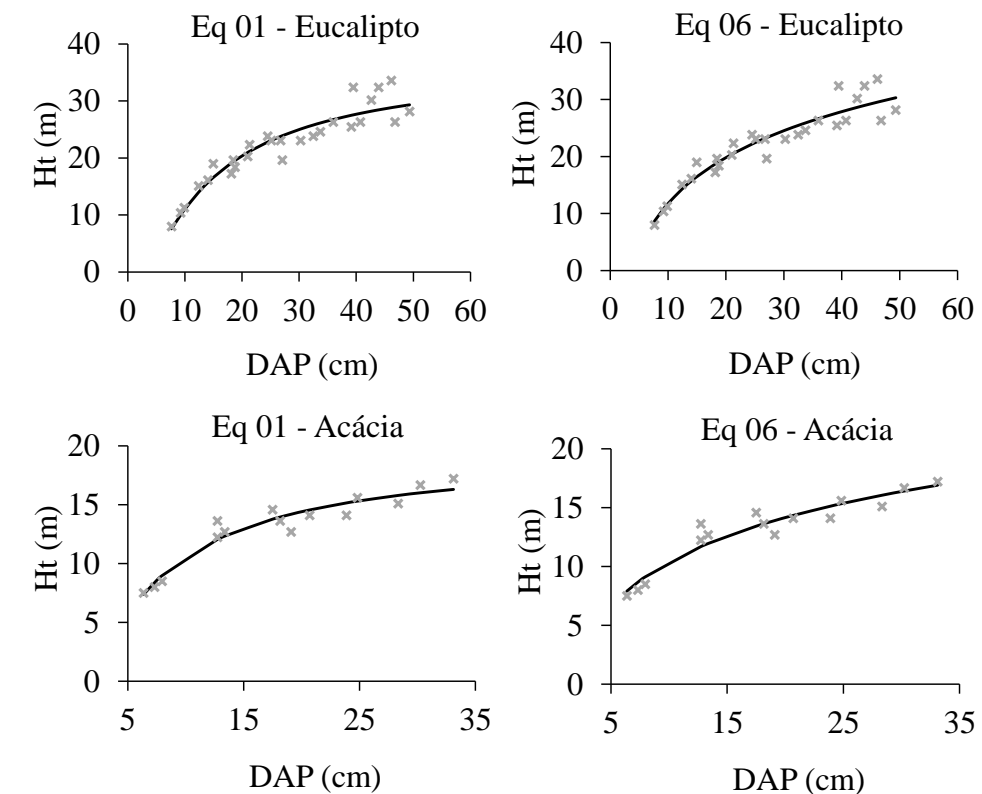

Figura 4. Tendências de altura estimada (Ht est.) versus altura observada (Ht obs.).

Figure 4. Estimated height versus observed height tendencies. 


\section{-V est. $\quad \times$ V obs}
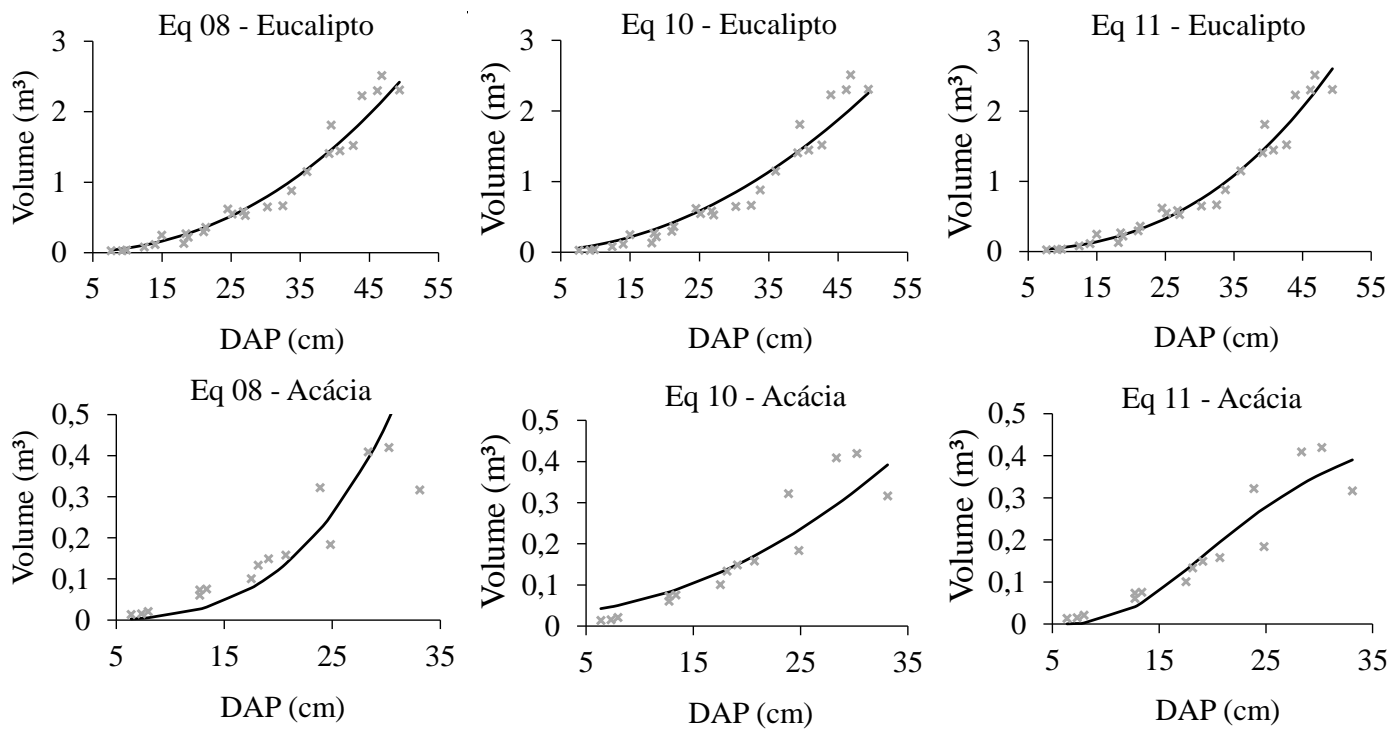

Figura 5. Tendências de volume estimado (V est.) versus volume observado (V obs.).

Figure 5. Estimated volume versus observed volume tendencies.

- dcc/DAP est. dcc/DAP obs

Eq 14 - Eucalipto

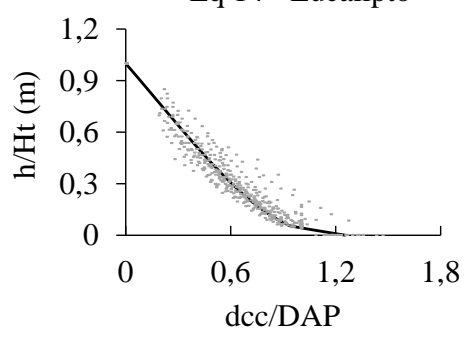

Eq 14 - Acácia

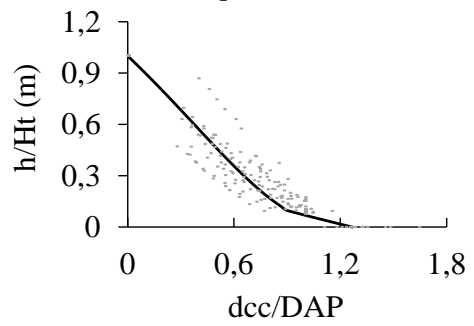

Eq 18 - Eucalipto

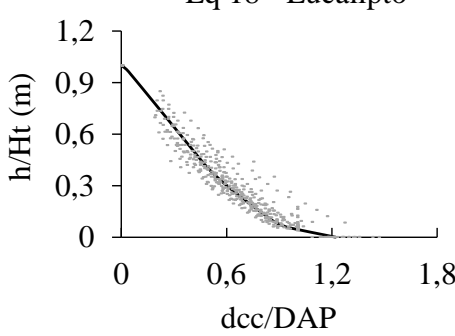

Eq 18 - Acácia

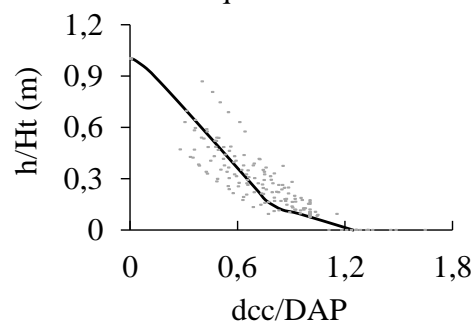

Eq 19 - Eucalipto

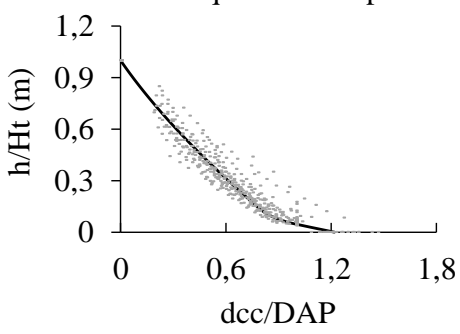

Eq 19 - Acácia

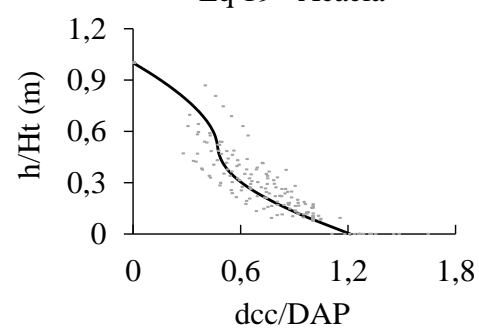

Figura 6. Tendências de perfil do fuste estimado (dcc/DAP est.) versus perfil do tronco observado (dcc/DAP obs.).

Figure 6. Estimated stem taper versus observed stem taper.

\section{CONCLUSÃO}

- Os modelos exponencial e semilogarítmico apresentam as melhores estatísticas para a estimativa da altura das árvores de eucalipto e acácia. 
- Os modelos de dupla entrada são superiores na estimativa de volume para as duas espécies e o modelo de Schumacher \& Hall o que apresentou as melhores estatísticas.

- Dentre os modelos volumétricos de simples entrada, destacam-se Brenac e Hohenadl \& Kreen que, apesar de inferiores aos modelos de dupla entrada, proporcionaram estatísticas satisfatórias.

- Para a descrição do perfil do tronco, os modelos segmentados geram melhores resultados que os não segmentados, com destaque para os modelos de Max \& Burkhart e Parresol. Dentre os não segmentados, destaca-se o modelo de Biging.

- Todos os modelos testados apresentam melhores ajustes para o eucalipto que para a acácia.

\section{AGRADECIMENTOS} financeiro

Os autores agradecem à Fundação de Amparo à Pesquisa do Estado de Minas Gerais, pelo apoio

\section{REFERÊNCIAS}

BARROS, D. A. de; MACHADO, S. A.; ACERBI JÚNIOR, F. W.; SCOLFORO, J. R. S. Comportamento de Modelos Hipsométricos Tradicionais e Genéricos para Plantações de Pinus oocarpa em Diferentes Tratamentos. Boletim de Pesquisa Florestal, Colombo, v. 45, n. 1, p. 03 - 28, 2004.

BARROS, P. L. C.; SILVA JÚNIOR, A. T. Equação de volume para árvores de uma floresta tropical densa no município de Anapú, oeste do estado do Pará, Amazônia Oriental. Revista de Ciências Agrárias, Belém, v. 51, p. 115 - 126, 2009.

BERGER, R.; SCHNEIDER, P. R.; FINGER, C. A. G.; HASELEIN, C. Efeito do espaçamento e da adubação no crescimento de um clone de Eucalyptus saligna Smith. Ciência Florestal, Santa Maria, v. 12 , n. 2, p. $75-87,2002$.

BINOTI, D. H. B.; SILVA, M. L. M.; LEITE, H. G.; MELIDO, R. C. N.; SANTOS, F. L. Descrição da distribuição diamétrica de sistemas agrossilvipastoris utilizando as funções Weibull e Hiperbólica. Revista Árvore, Viçosa, v. 36, p. 349 - 356, 2012.

BODDEY, R. M.; MACEDO, R.; TARRÉ, R. M.; FERREIRA, E.; OLIVEIRA, O. C.; REZENDE, C. P.; CANTARUTTI, R. B.; PEREIRA, J. M.; ALVES, B. J. R.; URQUIAGA, S. Nitrogen cycling in Brachiaria pastures: the key to understanding the process of pasture decline. Agriculture Ecosystems and Environment, v. 103, p. 389 - 403, 2004.

CAMPOS, J. C.; LEITE, H. G. (Ed). Mensuração Florestal: perguntas e respostas. 3(ed). Viçosa: Editora UFV, 2009. v. 1. 548 p.

COUTO, L.; BRANDI, R. M.; CONDÉ, A. R.; PAULA NETO, F. Influência do espaçamento no crescimento de Eucalyptus urophylla de origem híbrida, cultivado na região de Coronel Fabriciano, MG. Revista Árvore, Viçosa, v. 1, n. 2, p. 57 - 71, 1977.

FONTAN, I. C. I.; REIS, G. G.; REIS, M. G. F.; LEITE, H. G.; MONTE, M. A.; RAMOS, D. C.; SOUZA, F. C. Growth of pruned eucalypt clone in an agroforestry system in southeastern Brazil. Agroforestry Systems, v. 83, p. 121 - 131, 2011.

KVÅLSETH, T. O. Cautionary note about R². The American Statistican, v. 39, n. 4, p. 279 - 285, 1985.

MACEDO, R. L. G. Princípios básicos para o manejo sustentável de sistemas agroflorestais. Lavras: UFLA/FAEPE, 2000. 157 p.

MACHADO, S. A.; BARROS, D. A.; SCOLFORO, J. R.; ACERBI JÚNIOR, F. W. The effects of successive thinning on the hypsometric function for Pinus oocarpa stands. Floresta, Curitiba, v. 41, n. 2, p. 397 - 406, 2011.

MÜlleR, M. D.; NOGUEIRA, G. S.; CASTRO, C. R. T. de; PACIULlO, D. S. C.; ALVES, F. F.; CASTRO, R. V. O.; FERNANDES, E. N. Economic analysis of an agrosilvipastoral system for a mountainous area in Zona da Mata Mineira, Brazil. Pesquisa Agropecuária Brasileira, v. 46, p. 1148 $1153,2011$. 
NAIR, P. K. R. An introduction to agroforestry. Dordrecht: Kluwer Academic Publishers, 1993. 499 p.

NEVES, C. M. N.; SILVA, M. L. N.; CURI, N.; MACEDO, R. L. G.; MOREIRA, F. M. S.; D'ANDRÉA, A. F. Indicadores biológicos da qualidade do solo em sistema agrossilvipastoril no noroeste do estado de Minas Gerais. Ciência e Agrotecnologia, Lavras, v. 33, n. 1, p. 105 - 112, 2009.

NEVES, C. M. N.; SILVA, M. L. N.; CURI, N.; MACEDO, R. L. G.; TOKURA, A. M. Estoque de carbono em sistemas agrossilvopastoril, pastagem e eucalipto sob cultivo convencional na região noroeste do Estado de Minas Gerais. Ciência e Agrotecnologia, Lavras, v. 28, n. 5, p. 1038 - 1046, 2004.

NOGUEIRA, G. S.; LEITE, H. G.; REIS, G. G. MOREIRA, A. M. Influência do espaçamento inicial sobre a forma do fuste de árvores de Pinus taeda L. Revista Árvore, Viçosa, v. 32, p. 855 - 860, 2008.

PACIULLO, D. S. C.; CASTRO, C. R. T.; GOMIDE, C. A. M.; MAURÍCIO, R. M.; PIRES, M. F. Á.; MÜLLER, M. D.; XAVIER, D. F. Performance of dairy heifers in a silvopastoral system. Livestock Science, v. 141, p. 166 - 172, 2011.

SALLES, T. T.; LEITE, H. G.; OLIVEIRA NETO, S. N.; SOARES, C. P. B.; PAIVA, H. N.; SANTOS, F. L. Modelo de Clutter na modelagem de crescimento e produção de eucalipto em sistemas de integração lavoura-pecuária-floresta. Pesquisa Agropecuária Brasileira, Brasília, v. 48, p. 253 - 260, 2012.

SCHNEIDER, P. R.; COELHO, M. C. B.; ZANON, M. L. B.; FINGER, C. A. G.; KLEIN, J. E. M. Equações de volume para Eucalyptus dunnii Maiden, determinadas para a Depressão Central do Estado do Rio Grande do Sul. Ciência Rural, Santa Maria, v. 27, n. 3, p. 425 - 428, 1997.

SILVEIRA, D. P.; LEITE, H. G.; SOARES, V. P.; MELIDO, R. C. N. Classificação de árvores de eucalipto para postes em sistema agroflorestal. Revista Árvore, Viçosa, v. 35, p. 875 - 872, 2011.

SOUZA, C. A. M.; SILVA, G. F.; XAVIER, A. C.; CHICHORRO, J. F.; SOARES, C. P. B; SOUZA, A. L. Avaliação de modelos de afilamento segmentados na estimação da altura e volume comercial de fustes de Eucalyptus sp. Revista Árvore, Viçosa, v. 32, p. 453 - 463, 2008b.

SOUZA, C. A. M.; SILVA, G. F.; XAVIER, A. C.; MEDONÇA, A. R.; ALMEIDA, A. Q. Avaliação de modelos de afilamento não segmentados na estimação da altura e volume comercial de Eucalyptus sp. Ciência Florestal, Santa Maria, v. 18, p. 393 - 405, 2008a.

TONINI, H.; SCHWENGBER, L. A. M. Equações hipsométricas e volumétricas para Acacia mangium Willd em Roraima. Ambiência, Guarapuava, v. 2, p. 155 - 165, 2006.

VEIGA, R. A. A.; CARVALHO, C. M.; BRASIL, M. A. M. Determinação de equações de volume para árvores de Acacia mangium Wild. Cerne, Lavras, v. 6, p. 103 - 107, 2000.

XAVIER, D. F.; CARVALHO, M. M.; ALVIM, M. J.; BOTREL, M. A. Melhoramento da fertilidade do solo em pastagem de Brachiaria decumbens associada com leguminosas arbóreas. Pasturas Tropicales, v. 25, n. 1, p. $23-26,2003$. 
